\title{
Valoración en el cumplimiento de los objetivos en la cuatrimestralización de la UNED
}

\author{
GUISELLE HIDALGO MOLINA
}

(UNED, Costa Rica)

\begin{abstract}
RESUMEN: Las universidades se enfrentan a distintos desafios en la sociedad posmoderna debido a su complejidad, dinamismo y competitividad, que reclama la introducción de cambios que tienen implicaciones en el quehacer académico. Estas transformaciones, en algunos casos, significan un esfuerzo que involucra en forma integral a la organización. Precisamente, en este artículo se expone una situación semejante ocurrida en la UNED a raíz de la puesta en práctica de la modalidad de cuatrimestres en 1996. En su gestación se plantearon expectativas dirigidas a: la calidad de los programas académicos, la agilización del progreso del estudiante, el aprovechamiento de los recursos y la mejor dotación de recursos financieros producto del aporte estudiantil. El presente ensayo es una valoración de tales propósitos. Los de naturaleza cuantitativa se realizan a la luz del comportamiento de las series estadísticas respectivas; y para aquellos de carácter cualitativo se estudian sus variables asociadas.
\end{abstract}

\section{Educación a distancia - cuatrimestralización - series académicas - valoración de objetivos.}

\begin{abstract}
Universities face the challen ges of postmodern society due to its complexity, dynamism and competitivity that ctaims for chcznges which reflex th thw Acaaemia: In some cases, these transformations requiere an rn that involves all of the organization. This paper deals with such a situation as occured in UNED as o. consequence of the quatorization in 1996. It was expected to meet: quality of the academic program, an agile process of the progress of the student, better use of resources and of the finantial resources from student's. This assay values such purposes. Those of quantitative nature are analyzed thru doe behaviour of their serials and the qualitative ones after their associated variables.
\end{abstract}

\section{Distance Education - Quatonzatwre - Academic Sened - Obfectives assessment}




\section{INTRODUCCIÓN}

La Universidad Estatal a Distancia (UNED) de Costa Rica, a partir de 1996, transforma la periodicidad del proceso lectivo de una modalidad semestral a una cuatrimestral; así es que, a partir de este año, los estudiantes tienen la oportunidad de matricular un periodo adicional anual, y en cambio, disponen de menos tiempo en el periodo para cumplir con sus actividades de enseñanza y aprendizaje. A la fecha, se tiene experiencia con 13 cuatrimestres; éste es un tiempo suficiente como para poder valorar los resultados de su puesta en práctica. Para efectuar este estudio, se dispone de diferentes vías posibles a seguir: una de ellas es hacerlo frente a las expectativas que en un inicio motivaron este cambio en la duración de cada periodo académico, y este es, precisamente, el camino que orienta el presente trabajo.

Es comprensible que las motivaciones que inducen a una institución educativa a involucrarse en un cambio de tal naturaleza, sea producto de transformaciones de la sociedad que afectan su rol dentro de ella. En el caso de la UNED, este proceso sigue esa dirección, de forma tal que, se conceptúa dentro de la realidad que caracteriza las naciones posmodernas, las cuales al igual que en épocas anteriores, requieren profesionales con experticia en distintas áreas disciplinarias, pero se acentúan ciertos rasgos debido a algunas disposiciones impuestas por desafíos recientes que caracterizan dichas sociedades, en donde la competitividad y el dinamismo son componentes determinantes para lograr su desarrollo.

Entre los dístintivos que identifican al profesional, se destaca la necesidad de una actitud hacia la actualización ininterrumpida de los conocimientos disciplinarios, como condición para desempeñarse dentro de la actividad profesional (Escotet, 1992), así como de habilidades especializadas que faciliten la innovación y ofrezcan aportes significativos y pertinentes al nivel de crecimiento requerido (Drucker, 1995). Todo ello se espera lograr con una identificación social capaz de aportar al bienestar general dentro de su campo profesional.

En consecuencia, la respuesta educativa que corresponde a la educación superior debe ser entregada dentro de esquemas de calidad universal y en un tiempo prudencial, como para que se garantice la oportunidad de ese cuerpo profesional y permita la competitividad acorde con la tendencia globalizadora que caracteriza este mundo posindustrial basado en el conocimiento.

Circunscrito a este contexto social, la implementación de esta modalidad cuatrimestral pretende responder al interés de brindar un servicio educativo adecuado a tales exigencias, en virtud de la posibilidad que surge para cambiar y actualizar la oferta académica, de forma tal que, entre otras cosas, promueva una revisión y una adaptación del curriculum, la introducción de innovaciones académicas, la actualización del paquete didáctico y una mejor aprovechamiento de los recursos humanos, tecnológicos, físicos y financieros disponibles para el quehacer académico. Aunado a esto, se plantea también la opción de acortar la permanencia del estudiante dentro de la carrera y asimismo, poder lograr una mayor generación de recursos propios. De igual forma, el 
estudiante tiene la oportunidad de insertarse a más temprana edad en el mercado laboral. Todo ello coadyuvaría a mejorar la imagen institucional y a facilitar una mayor absorción de la población estudiantil del país.

Ante estas expectativas, el presente ensayo pretende valorar aquellos objetivos que están ligados a un enfoque cuantitativo a la luz del comportamiento de algunas series estadísticas claves y a buscar un acercamiento con aquellos que, aunque Fuesen de naturaleza cualitativa, pueden reflejar, en forma indirecta, algún comportamiento de variables educativas básicas. Además, también se introduce una análisis cualitativo focalizado que permite valorar ciertos aspectos de calidad académica en cuatro ejes programáticos de la carrera que concentra la mayor población estudiantil y que es formadora de los educadores en 1 y II ciclos del país.

Por lo anterior, el presente trabajo se estructura en tres secciones: en la primera se valoran estos objetivos iniciales con base en series estadísticas de las variables asociadas disponibles en la institución para el periodo 1994-1999 (hasta el segundo cuatrimestre), que comprende en total 15 periodos académicos. En una segunda sección se estudian los efectos que la reducción del periodo pudo haber ocasionado, estos no necesariamente previstos en el proyecto gestor, tal como la respuesta a la evaluación de los aprendizajes y la motivación hacia la permanencia o la deserción. Finalmente, una última sección, comprende algunas implicaciones académicas de la cuatrimestralización, focalizadas en la carrera de Ciencias de la Educación en 1 y II Ciclos, donde se incorporan elementos cualitativos y se revisa la situación de cuatro de sus materias ejes, en aspectos que se prevén de antemano en el acuerdo del Consejo Universitario, sesión No. 1171-95, Art. II, inciso 2, tales como las adaptaciones curriculares, las evaluaciones de aprendizaje, la reducción de tutorías, entre otras.

Esta parte se incorpora como un intento por reflejar las posibles repercusiones en la calidad académica, situación que no es posible analizar con solo indicadores cuantitativos. Sin duda ésta tiene gran relevancia institucional que justifica de por sí su consideración en cualquier estudio relacionado. Por ello se hace, en este sentido, la mención que aparece en el Proyecto de Cuatrimestralización (1992), donde se señala una experiencia similar ocurrida 20 años antes en la Universidad de Costa Rica. Ahí se indica que: "los docentes concordaron en que el sistema de cuatrimestres permite una mejor utilización de los recursos y reduce la duración de las carreras, aunque Fueron del criterio de que la calidad académica se redujo" (UNED, 1992, p.10).

\section{OBJETIVOS DEL PROCESO CUATRIMESTRAL}

De las consideraciones anteriores se derivan los cuatro objetivos que este proceso pretende alcanzar:

1. Aceleramiento del progreso del estudiante en su carrera

2. Mejoramiento de la competitividad institucional

3. Disposición de mayores recursos financieros propios

4. Mejor aprovechamiento de los recursos de la Universidad 
Estos objetivos son tanto de naturaleza cuantitativa como cualitativa. El primero y el tercero son de naturaleza numérica y en consecuencia, la valoración es con un enfoque cuantitativo. El segundo de ellos sólo puede ser evaluado en forma indirecta con este tipo de análisis y en ese sentido queda analizado parcialmente. Y con respecto al último objetivo es aplicable más bien un análisis costo- efectividad que combine elementos cualitativos y cuantitativos que por su dimensión no se contempla en este trabajo.

\section{METODOLOGÍA}

Para llevar a cabo el análisis estadístico se utilizaron series numéricas del periodo 19941999 (hasta el $2^{\circ}$ cuatrimestre); es decir, este estudio comprende dos años del periodo semestral y más de 3 años del periodo cuatrimestral, que da un total de 4 semestres y 11 cuatrimestres. Los datos seleccionados están disponibles en los documentos oficiales de la Universidad y representan fuentes primarias de información. De ahí que se eligieron los anuarios estadísticos y los sistemas de información institucional. De manera que para ciertas series de interés de este estudio que no estuvieran disponibles en los anuarios o que se obtiene con ello, una mayor confiabilidad, se acude a la unidad generadora para que ésta fuera elaborada en la medida de las posibilidades. Asimismo, se utilizaron algunos datos contenidos en los informes de labores del Rector.

Por último, y con el interés de conocer la incidencia de la cuatrimestralización en la situación particular de los programas académicos de la UNED, como se indica arriba, se selecciona la carrera Ciencias de la Educación en Iy II Ciclos y se eligen cuatro de sus ejes programáticos. Este estudio focalizado en esta carrera comprende una valoración cualitativa, producto de la entrevista efectuada a la encargada de ese programa académico y a los encargados de las cátedras a que estos ejes pertenecen. Si bien por razones de tiempo no se profundiza con otros agentes importantes del proceso como son los estudiantes, los tutores, las autoridades académicas, ni se abarcan todos sus ejes programáticos, sí se pretende delinear un camino para que en el futuro se ampife esta valoración a tales sectores, así como también se contemplen otros cursos y otras carreras que ofrece la Universidad.

En otras palabras, se propone una metodología para conocer las implicaciones que a nivel académico tuvo la cuatrimestralización y tener la opción de realizar los ajustes pertinentes gradualmente con algún criterio de priorización. Entre ellos se pueden mencionar: la población estudiantil atendida, la relevancia de la carrera en la sociedad, la contribución en el fortalecimiento de la imagen institucional, la atención a las poblaciones inherentes al sistema educativo a distancia, la incorporación de sectores sociales preferenciales, entre otros aspectos que pueden definirse.

Se hace notar que este artículo es elaborado con base en un estudio denominado: Evaluación del proceso de cuatrimestralización a la luz de algunas series estadísticas, realizado en octubre de 1999 por la autora y que tiene el apoyo profesional de la Dra. Guiseppa D'Agostino S.

\section{CUMPLIMIENTO DE OBJETIVOS}

\subsection{ACELERAMIENTO DEL PROGRESO DEL ESTUDIANTE EN SU CARRERA}


Para poder valorar el primer objetivo planteado sobre el grado de avance académico de los estudiantes, muy posiblemente, una de las variables más indicadas es el plazo promedio para obtener el título en las distintas cohortes del periodo considerado, así como también, otra variable que se ajusta al propósito es: el número de estudiantes graduados por cada "n" años según grado académico. No obstante, la obtención de los datos de esta serie, por diversas razones, no están disponibles y en su lugar, se han elegido las tres variables siguientes: el número de materias promedio matriculadas al año, el rendimiento promedio por periodo académico y el número de graduados al año.

En virtud de la oportunidad de disponer de un periodo lectivo adicional en el año, el estudiante puede lograr un avance mayor en sus estudios universitarios. No obstante, su progreso a un ritmo mayor sólo se verá reflejado, si éste tiene la posibilidad de mantener o mejorar su rendimiento académico dentro de las nuevas condiciones impuestas por la cuatrimestralización. De ahí que es importante determinar también, los resultados de su rendimiento académico; es por esta razón por la que se elige la segunda variable indicada. Además, se espera también obtener un incremento en el número de graduados en los años posteriores a la cuatrimestralización dada a la expectativa de que el estudiante logra finalizar más rápido su carrera universitaria, de ser así, esta valoración se debiera reflejar en la puesta en operación del proceso cuatrimestral.

\section{COMPORTAMIENTO DE LA MATRÍCULA POR PERIODO ACADÉMICO}

Cuadro No. 1 No. promedio de asignaturas matriculadas por periodo académico (19941999) 


\begin{tabular}{|ll|}
\hline Año & Promedio de materias \\
\hline I994 & 5.35 \\
\hline I & 2.72 \\
\hline II & 2.63 \\
\hline 1995 & 5.43 \\
\hline I & 2.78 \\
\hline$\|$ & 2.65 \\
\hline 1996 & 6.55 \\
\hline I & 2.24 \\
\hline II & 2.14 \\
\hline III & 2.17 \\
\hline 1997 & 6.82 \\
\hline I & 2.29 \\
\hline II & 2.17 \\
\hline III & 2.36 \\
\hline 1998 & 7.06 \\
\hline I & 2.34 \\
\hline II & 2.35 \\
\hline III & 2.37 \\
\hline 1999 & \\
\hline I & 2.35 \\
\hline II & 2.30 \\
\hline
\end{tabular}

Fuente: Oficina de Registro, octubre 1999

Del cuadro anterior se desprende que, al entrar en operación el sistema de cuatrimestres, el estudiante matncula ligeramente menos asignaturas en promedio por periodo académico; sin embargo, con el tercer periodo, éste tiene la oportunidad de aumentar el número de materias que cursa al año. Así es que, en 1996 matricula una asignatura más en promedio con respecto al año anterior. En los siguientes años, se observa un incremento creciente que puede ser un reflejo de la adaptación del estudiante a las exigencias de la nueva modalidad, situación que se puede corroborar en el cuadro No. 1. El número promedio de materias inscritas por año asciende de 5.43 en 1995 a 6.55 en 1996, a 6.82 en 1997 y a 7.06 en 1998 (ver gráfico No. 1). Esta serie muestra que hay un aumento en el número de materias que matricula por año el estudiante.

Materias matriculadas en promedio al año 


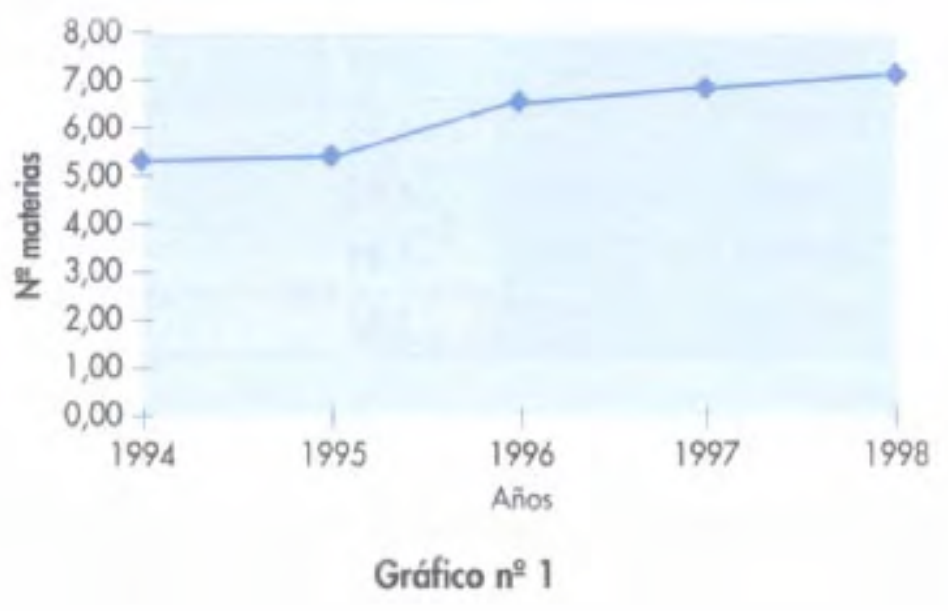

Para esta reducción pudo haber mediado razones económicas, o consideraciones sobre el esfuerzo adicional implicado o el interés de conservar el mismo rendimiento y así con ello, no perder, por ejemplo, el incentivo de una beca estudiantil. Pese a lo anterior, esto no implica el hecho de que, eventualmente, un sector de estos estudiantes haya aprovechado la oportunidad para aumentar el ritmo de avance por periodo y que esto se refleje en otros resultados, como pueden ser, un mayor número de graduados por año. Esto es así, dado que, con estos datos, se refieren a la situación global de la población estudiantil, por ende, no discrimina en sectores específicos.

\section{COMPORTAMIENTO DEL RENDIMIENTO ACADÉMICO}

Cuadro No. 2 Rendimiento promedio por periodo académico (1994-1999) 


\begin{tabular}{|l|l|}
\hline Periodo Académico & $\begin{array}{l}\text { Rendimiento promedio } \\
\text { de estudiantes: }\end{array}$ \\
\hline $1994-1$ & 5.45 \\
\hline $1994-2$ & 5.72 \\
\hline $1995-1$ & 5.60 \\
\hline $1995-2$ & 5.63 \\
\hline $1996-1$ & 5.84 \\
\hline $1996-2$ & 5.82 \\
\hline $1996-3$ & 6.29 \\
\hline $1997-1$ & 5.95 \\
\hline $1997-2$ & 6.11 \\
\hline $1997-3$ & 6.12 \\
\hline $1998-1$ & 5.91 \\
\hline $1998-2$ & 5.96 \\
\hline $1998-3$ & 5.92 \\
\hline $1999-1$ & 5.85 \\
\hline $1999-2$ & 5.81 \\
\hline
\end{tabular}

*No se presentó $(\mathrm{NSP})=5.0$

Fuente: Oficina de Registro, octubre 1999

En el cuadro No. 2 se presenta el rendimiento promedio de los estudiantes por curso: incorpora a los que finalizaron todas las evaluaciones previstas, así como aquellos que se retiraron antes de finalizar el periodo lectivo sin presentar justificación; es decir, en esta última situación se incluyen además los denotados NSP (No se presentó). Hay que recordar que en los sistemas a distancia, esa ocurrencia suele ser Frecuente y es un tipo de deserción. Se observa que la cuatrimestralización no afectó en sentido negativo, resultado que es comprensible ante la realidad de que el estudiante hace una mejor distribución de la carga académica. Así, se nota un ligero progreso, pues pasa de un rendimiento promedio entre 5.40 a 5.60 para los años 1994-1995, a un intervalo superior de 5.80 a 6.30 para el periodo 1996-1999 (ver gráfico No. 2). Esta mejora en el rendimiento se detecta a pesar de la incidencia importante que tienen los NSP en el cálculo, dado que en la mayoría de los casos este abandono es del 50\%, y aún así, se obtiene incremento en el rendimiento promedio en la nueva modalidad. Es decir, la moda estadística es de 5.00 por la incidencia de estos NS?, en una distribución asimétrica positiva, donde el intervalo para el periodo cuatrimestral supera al intervalo semestral pese al efecto reductivo que ejercen los NSP; de ahí que se puede afirmar que hay un aumento en el rendimiento académico para la nueva modalidad. 
Rendimiento promedio de estudiantes

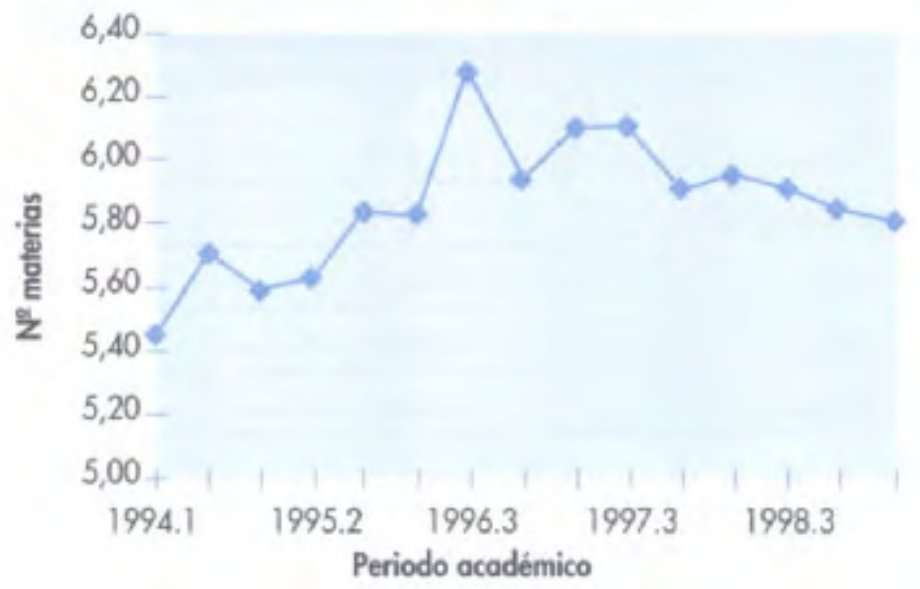

Gráfico n 2

Comportamiento de graduados

En cuanto a las repercusiones de la cuatrimestralización en el comportamiento de los graduados de estudios, se observa una marcada diferencia al entrar este sistema en vigencia. En el cuadro No.3, puede notarse este efecto. El número de graduados en el año 1996 se incrementó casi en un 50\% con respecto a los años previos y después, al entrar el año de 1997, se observa una estabilidad en el comportamiento para este sector del periodo cuatrimestral. De ahí que, luego del gran salto inicial, se nota un nuevo comportamiento caracterizado por la capacidad institucional de generar una cantidad mayor a los 1600 graduados por año, contra los casi 900 graduados del sistema semestral anterior. Para el año 1998 continúa la tendencia creciente; así, el nivel de graduados tiene un incremento importante de $35 \%$.

Estos resultados pueden deberse, en parte, al impacto provocado por las nuevas medidas tomadas respecto a la diversificación de opciones para el trabajo de graduación, que entraron en vigencia en ese periodo y se exponen en el acuerdo 1181-95, Art. III, inciso 3 del Consejo Universitario.

Cuadro No. 3 No. de graduados por grado académico (1994-1999)

\begin{tabular}{|c|c|c|}
\hline Periodo Académico & Graduodos & $\%$ \\
\hline 1994 & 828 & \\
\hline 1995 & 898 & 8.5 \\
\hline 1996 & 1678 & 46.5 \\
\hline 1997 & 1651 & $-0,02$ \\
\hline 1998 & 2236 & 35.4 \\
\hline 1999 & 1992 & -10.9 \\
\hline
\end{tabular}


Fuente: Anuarios estadísticos 1996, 1997

Oficina de Registro, octubre 1999

Dadas las implicaciones tan notables observadas en la cantidad de graduados del sistema actual, interesa profundizar más en este aspecto; de ahí que, en el cuadro No. 4, se registran estos datos a nivel del grado académico otorgado. Así, al entrar el proceso de cuatrimestres en 1996, se observa un incremento importante en los estudiantes que alcanzaron su título a nivel de diplomado, cerca del 65\%. Más acentuada aún es la variación porcentual en aquellos que obtuvieron grados mayores como en el bachillerato y en la licenciatura, puesto que en estos últimos casos se observa una duplicación en el número de estudiantes que lograron tales grados académicos. En el año siguiente del periodo estudiado se nota, otra vez, una estabilización.

Los incrementos dentro del periodo de cuatrimestralización tienen un comportamiento uniforme, excepto en el caso de licenciados, donde todavía se observa un incremento importante de más del $50 \%$. Este último resultado hace pensar que la flexibilización en los requisitos de graduación mencionada ha permitido que una población rezagada esté efectivamente logrando finalizar sus estudios, así que, una vez satisfecha esta población, posiblemente, el comportamiento tienda a normalizarse igual que en los otros grados académicos.

En conclusión, independientemente de los factores que lo hayan generado (cuatrimestralización- diversificación de las opciones para graduarse) se observa un salto marcado en la serie de graduados por título obtenido al entrar la nueva modalidad, para luego alcanzar uniformidad en los años siguientes. No obstante, se espera que este nivel tienda a aumentar con el tiempo por los dos argumentos citados: un aumento en las materias cursadas al año y laflexibilización de los requisitos de graduación.

Cuadro No. 4 Graduados por grado académico (1994-1999)

\begin{tabular}{|c|c|c|c|c|c|c|c|c|}
\hline Anio & Tennico & $\begin{array}{l}\text { Tecrico de } \\
\text { Extensión }\end{array}$ & Diplomodo" & Profesor" & Bochiller" & Bicenciodo: & Móster" & Total \\
\hline 1994 & 66 & & 402 & & 265 & 95 & & 828 \\
\hline \multicolumn{9}{|l|}{$\Delta \%$} \\
\hline 1995 & 91 & & 415 & & 239 & 103 & & 848 \\
\hline$\Delta \%$ & & & 3.2 & & -9.8 & 8.4 & & \\
\hline 1996 & 79 & 191 & 681 & 11 & 498 & 212 & 6 & 1678 \\
\hline$\Delta x$ & & & 64.0 & & 108.4 & 105.8 & & \\
\hline 1997 & 12 & 77 & 667 & 17 & 545 & 329 & 4 & 1651 \\
\hline$\Delta \%$ & & & -26 & & 9.4 & 55.2 & & \\
\hline 1998 & 53 & 115 & 839 & 17 & 639 & 563 & 10 & 2236 \\
\hline$\Delta \%$ & & & 25.8 & & 17.3 & 71.1 & & \\
\hline $1999 *$ & 0 & 46 & 746 & 19 & 684 & 493 & 4 & 1992 \\
\hline$\Delta \%$ & & & -11.1 & & 7.0 & .12 .4 & & \\
\hline Tat & & & & & & & & \\
\hline
\end{tabular}

* Estudios formales 
** Dato preliminar

Fuente: Estadísticas 1992-1996

Anuario estadístico 1997

Oficina de Registro, octubre 1999

Comportamiento de la oferta académica

En relación con la oferta de asignaturas por periodo académico, se hicieron consideraciones previas al inicio de la modalidad cuatrimestral. En primer ia se establece el propósito de que cada materia sea ofrecida por lo menos z al año, Por otro lado, se decide disminuir el número de éstas en oferta en enoao académico y así poder reducir los costos de operación, y facilitar la ción de tutorías presenciales, la administración de los exámenes, entre rentajas señaladas en ese entonces.

También se considera que con la oferta cuatrimestral hay una mejor rotación nventario de materiales didácticos, lo que permite, a su vez, una mayor opord de actualización del paquete instruccional.

Ahora bien, a partir de estas medidas se concluye que el estudiante tiene sóposibilidad real de avanzar más rápido en su carrera universitaria si la mase ofrece en los tres cuatrimestres, pues de otra forma, queda en una circunstancia parecida a la modalidad semestral o en una condición más desventajosa si sólo se ofrece una vez al año. Además, en este último caso existe el riesgo de que se formen concentraciones anuales por la acumulación de estudiantes en espera de la oportunidad de cursar la asignatura.

Esta circunstancia, también puede afectar la cantidad de materias que puede matricular al cuatrimestre, y en ese sentido, la disminución del ritmo de matrícula con el nuevo sistema que fue referida al inicio de la presente sección puede estar más bien relacionada con una menor oportunidad de la oferta académica, lo que en definitiva afecta el aceleramiento de su progreso académico. Así que, esta medida no está inscrita en la dirección de facilitar una reducción en la carrera, sino en favorecer una distribución mejor de su carga al año, lo que eventualmente puede contribuir a mejorar su rendimiento. En ese mismo sentido, esta reducción de oferta por periodo limita la posibilidad prevista de una mayor circulación en los inventarios de libros.

No obstante, otras medidas alternativas se tomaron para compensar la falta de oferta mencionada; así, se decide abrir para cualquier materia la oportunidad para optar por su acreditación por la vía de suficiencia ampliada a todas las asignaturas disponibles. Esta medida estuvo vigente hasta el primer cuatrimestre de 1999, cuando se decide eliminarla por la dificultad de elaborar los instrumentos 1 de evaluación y se deja la suficiencia restringida a solo las materias en oferta en el periodo académico. A raíz de esta situación, se limita la opción por suficiencia para cualquier asignatura que está fuera de la oferta de ese periodo, pero queda todavía habilitada la posibilidad del examen centralizado. A esta última opción sólo pueden optar aquellos estudiantes que les falte cuatro o menos asignaturas para concluir su programa de estudios y para ser autorizada se requiere realizar un trámite poco ágil y sumamente engorroso para el encargado de programa y de cátedra. Esta situación se complica cuando el número de estudiantes que lo solicitan es considerable. Además, el examen comprensivo puede dificultar su aprobación, de manera que el estudiante en estas circunstancias tiene 
menos posibilidades de mejorar el progreso de su programa académico y de su aprendizaje.

Esta situación se mejora a partir de 1998 cuando se realiza una planificación de la oferta basada en la experiencia adquirida con la modalidad, se efectúan proyecciones para los dos años siguientes y esto permite darle oportunidad al estudiante para organizar su matrícula con suficiente antelación.

En el cuadro No. 5 se presenta la oferta anual y por periodo académico. Se observa que la medida prevista de reducción de la oferta en cuatrimestres se pone efectivamente en práctica para los años de 1996 y 1997. Luego, en 1998, con los cambios en su planificación, se observa la recuperación del nivel semestral (ver gráfico No. 3). Así se incrementa la cantidad de materias que ahora se ofrecen en los tres cuatrimestres, y con ello, la posibilidad de cursar más materias al cuatrimestre. Tal situación es consistente con el primer objetivo planteado.

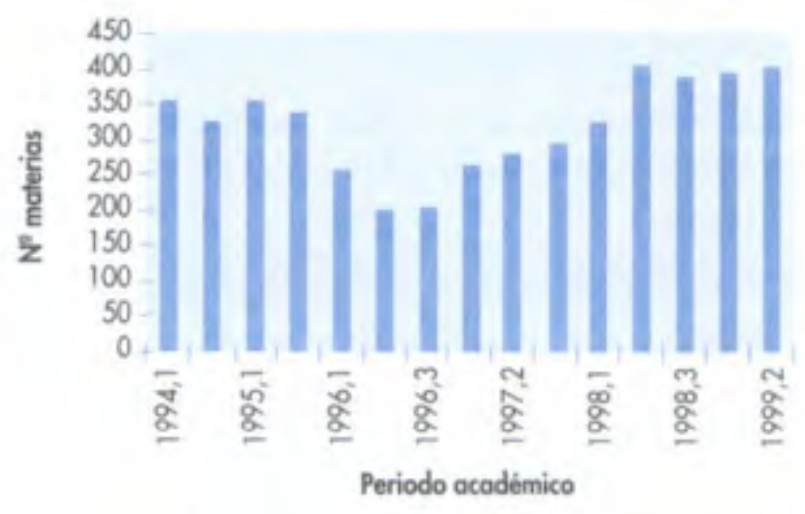

Gráfico no. 3 No. de materias en oferto por periodo académico

En conclusión, frente al primer objetivo analizado, los resultados son positivos. Se observa que efectivamente existe un sector estudiantil que ha podido aligerar su periodo de graduación. Por otra parte, los estudiantes no incrementaron las asignaturas matriculadas en promedio en el cuatrimestre, sino que atienden una menor cantidad de ellas por periodo académico; se puede suponer que existe otro sector que mantiene su ritmo de avance anual. Pese a esto, esa mejor repartición de su carga académica anual puede haber contribuido a que el estudiante mejore su rendimiento académico. Así mismo, es importante tener presente también la calidad académica ofrecida, puesto que a la par de un progreso satisfactorio y de un rendimiento mayor, se requiere lograr un nivel de excelencia suficiente para la formación de profesionales competentes y para el alcance de un nivel de competitividad institucional dentro sector educativo. 
Cuadro No.5 No. de materias en oferta por periodo académico

\begin{tabular}{|c|c|}
\hline Periodo Acodémico & No. materias en oferta \\
\hline 19941 & 356 \\
\hline 1994 || & 324 \\
\hline \multicolumn{2}{|l|}{1994} \\
\hline 19951 & 354 \\
\hline 1995 ॥ & 334 \\
\hline \multicolumn{2}{|l|}{1995} \\
\hline 19961 & 253 \\
\hline $1996 \|$ & 196 \\
\hline 1996 III & 200 \\
\hline \multicolumn{2}{|l|}{1996} \\
\hline 19971 & 257 \\
\hline 1997 ॥ & 276 \\
\hline 1997 III & 292 \\
\hline \multicolumn{2}{|l|}{1997} \\
\hline 19981 & 321 \\
\hline 1998 ॥I & 402 \\
\hline 1998 III & 385 \\
\hline \multicolumn{2}{|l|}{1998} \\
\hline 19991 & 390 \\
\hline 1999 ॥ & 402 \\
\hline \multicolumn{2}{|l|}{1999 III } \\
\hline 1999 & \\
\hline
\end{tabular}

Fuente: Oficina de Registro, octubre 1999

\subsection{MEJORAMIENTO DE LA COMPETITIVIDAD INSTITUCIONAL}

En relación con el segundo objetivo, cabe preguntar: ¿qué tan atractiva pudo resultar la oferta de cuatrimestres para la población estudiantil del país? y esto, considerado como condicionante, aparte de la motivación ya existente en la modalidad de enseñanza a distancia. Sin duda, su mejor conocimiento requiere de una valoración del mercado educativo. Pese a esto, también es cierto que esta situación puede implicar un incremento de la admisión y de la matrícula estudiantil anual. Como un intento de acercamiento a la valoración de esta inquietud, se estudia sus efectos en estas dos variables académicas claves para el periodo considerado.

Comportamiento de la admisión

Con respecto a la admisión, se nota en el cuadro No. 6, que los dos primeros cuatrimestres en cada año del periodo estudiado, tienen un comportamiento similar a los dos semestres de los últimos años del sistema anterior que se han considerado en este análisis. Además, el tercer cuatrimestre de cada año tiene una admisión menor a los dos primeros, y esta cantidad, prácticamente, corresponde con el incremento observado en el número de los admitidos anual al entrar el nuevo sistema cuatrimestral. 
Esto hace pensar que, aparentemente, los estudiantes nuevos se incrementaron por la oportunidad de tener un nuevo periodo lectivo al año. Esto se reafirma aún más, con el hecho de que la UNED ofrece esa oportunidad adicional, justamente, en la última semana de agosto, cuando las restantes instituciones de educación superior estatales han finalizado para ese año, sus respectivos periodo de admisión. Sobre esta referencia, cabe recordar que la Universidad Nacional inició su sistema trimestral en forma generalizada a partir de febrero de 1999. Ante esta situación, es difícil determinar cuánto de este incremento se debe a la buena impresión que pudo producir la posibilidad de cursar una carrera universitaria de menor duración, contra la realidad de ser ésta la única opción de iniciar estudios en una universidad estatal a partir de esa época del año.

Independientemente de la situación anterior, se nota un aumento anual importante en estudiantes nuevos para el sistema de cuatrimestres con respecto al sistema semestral. En el año 1995 hubo un incremento anual del 13\% y en 1996, esta variación fue del 25\%. Esto significa, que por cada 8 estudiantes nuevos en 1994 se tienen cerca de 9 estudiantes más en 1995, mientras que, por cada 4 estudiantes de éstos en 1995, se admitieron aproximadamente 5 estudiantes en el año 1996. De manera que se pasó de admitir 4968 a 6189 estudiantes de nuevo ingreso en esos dos años respectivamente. Pese a este salto, para los restantes años se observan pequeños incrementos anuales que no superan el $8 \%$, lo que muestra, una vez más que, se da uniformidad dentro del periodo cuatrimestral.

Cuadro No. 6 No. de estudiantes admitidos y matriculados por periodo académico (1994-1999)

\begin{tabular}{|c|c|c|c|c|c|}
\hline $\begin{array}{l}\text { Periodo } \\
\text { Acodémico }\end{array}$ & Admision & $\Delta \%$ & \% Admifidos & Matricula & $\stackrel{\Delta \%}{M}$ \\
\hline 19941 & 2283 & & 19.5 & 11706 & \\
\hline $1994 \|$ & 2022 & & 17.7 & 11425 & \\
\hline 1994 & 4305 & & & 23131 & \\
\hline 19951 & 2795 & & 20.8 & 13428 & \\
\hline 1995 II & 2173 & & 17.2 & 12629 & \\
\hline 1995 & 4968 & 13.3 & & 26057 & 12.6 \\
\hline 19961 & 2453 & & 19.8 & 12371 & \\
\hline 1996 II & 2155 & & 17.7 & 12144 & \\
\hline 1996 ill & 1581 & & 13.4 & 11777 & \\
\hline 1996 & 6189 & 24.6 & & 36292 & 39.3 \\
\hline 19971 & 2794 & & 19.9 & 14036 & \\
\hline 1997 ॥ & 2006 & & 14.6 & 13763 & \\
\hline 1997 IIII & 1600 & & 127 & 12595 & \\
\hline 1997 & 6400 & 3.4 & & 40394 & 11.3 \\
\hline 19981 & 2918 & & 20.5 & 14264 & \\
\hline $1998 \|$ & 2229 & & 16.5 & 13516 & \\
\hline 1998 III & 1723 & & 11.0 & 15641 & \\
\hline 1998 & 6870 & 7.3 & & 41321 & 2.30 \\
\hline 19991 & 3464 & & 22.3 & 15544 & \\
\hline $1999 \|$ & 2262 & & 15.6 & 14461 & \\
\hline
\end{tabular}


Fuente: Oficina de Registro, octubre 1999

Comportamiento de la matrícula

Ahora bien, en este mismo cuadro, y con respecto a la matrícula, se nota que, como era de esperar, hubo un incremento anual mayor al iniciar la cuatrimestralización. En términos relativos este aumento fue de $39 \%$, mientras que en el último año del sistema semestral este incremento era de $13 \%$. Luego, y en forma similar a lo observado para la admisión, se alcanza estabilidad dentro del periodo cuatrimestral estudiado (19961999), donde se observa un incremento pequeño que no supera el $12 \%$. Cabe aclarar que el total anual indicado en el cuadro se refiere a la cantidad de matrículas y no al número de estudiantes matriculados en el año, puesto que la primera variable se aplica más al aspecto analizado.

Por otro lado, la matrícula ocurrida en los dos primeros cuatrimestres del año, no desestimula la matrícula en el tercer cuatrimestre, todo lo contrario, los estudiantes aprovecharon la oportunidad de un nuevo periodo lectivo para avanzar en su programa de estudios, aunque se recordará la observación de que, no se logra sostener el ritmo de avance por periodo académico pretendido en relación con el sistema semestral, puesto que el número promedio de material matriculadas por cuatrimestre disminuyó ligeramente.

Con respecto al cumplimiento del segundo objetivo, se puede concluir que el periodo cuatrimestral produjo un aumento en forma significativa en la cantidad de estudiantes admitidos y matriculados durante el año. No obstante, los datos disponibles dificultan precisar si esta variación fue motivada por una impresión favorable en el estudiante hacia el esfuerzo institucional de agilización en su servicio académico. Pese a lo anterior, se puede afirmar que estas cifras no contradicen el supuesto derivado de este objetivo.

En el gráfico No. 4 se muestra el comportamiento de las dos series que configuran la población estudiantil.

Admisión y matrícula anual

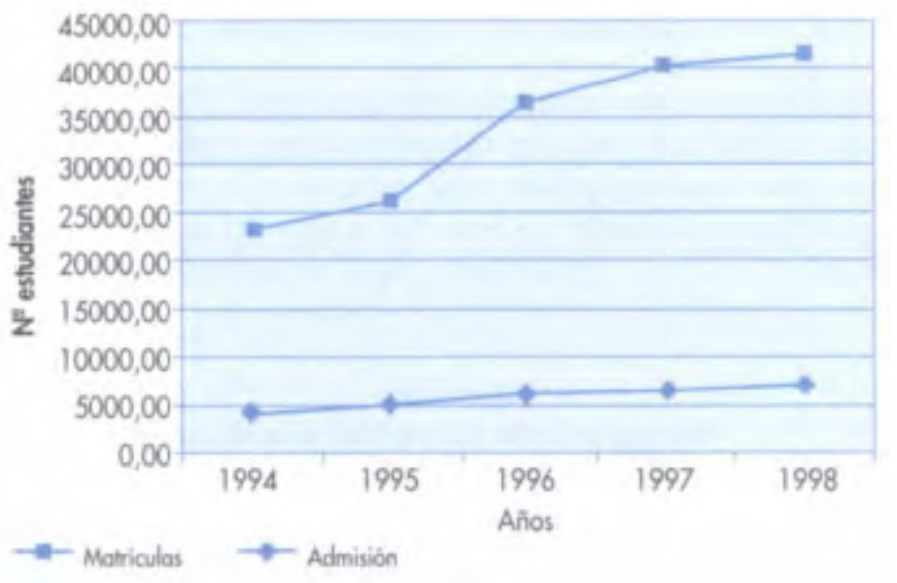

Gráfico no 4 


\subsection{DISPOSICIÓN DE MAYORES RECURSOS FINANCIEROS PROPIOS}

Para el cumplimiento del tercer objetivo ligado a una mayor disponibilidad financiera institucional producto de la cuatrimestralización, en esta sección del documento se analiza el comportamiento de los recursos generados internamente durante el lapso considerado; esta valoración obviamente, no comprende los Costos adicionales que implica el nuevo periodo lectivo anual, así que es tan solo una vista parcial de las implicaciones de tipo financiero.

La composición de los ingresos propios no ha tenido cambios importantes con la cuatrimestralización. En el cuadro No. 8 se observa que el crecimiento de estos recursos en 1994-1995 es alrededor del 24 y $25 \%$ y en el periodo 1996-1997 tiene un aumento leve, poco más del $25 \%$ a cerca del $27 \%$. La razón de tal comportamiento puede estar en la situación comentada de que el estudiante matricula en promedio menos materias por periodo académico, a raíz, posiblemente, de una oferta reducida o del peso adicional que significa la modalidad cuatrimestral.

Así, como se hace mención antes, en 1996 el estudiante únicamente matricula en promedio una materia más que en 1995; tal vez esta situación tienda a variar y permita un incremento más significativo cuando, por un lado, el estudiante se adapte mejor a las nuevas exigencias académicas y económicas de la nueva modalidad, y por otro lado, la Universidad logre ampliar su oferta académica, entre otros aspectos que se recomiendan al final del documento.

Por otra parte, el comportamiento en el superávit anual, no es un factor que afecte el porcentaje de ingresos propios, puesto que, aún si se asume para $1996 \mathrm{el} \mathrm{mismo} \mathrm{nivel} \mathrm{de}$ 1995, este indicador si acaso alcanza el $27 \%$.

Ahora bien, el porcentaje de ingresos propios en el 1998 tiene un aumento ligeramente diferente al indicado para los años anteriores, es del $29 \%$ con respecto a los ingresos totales. Esta situación puede estar relacionada con la apertura de la oferta académica que se observa a partir de este año, a raíz del esfuerzo realizado para un mejor planeamiento, y de esta manera se le da al estudiante una mejor oportunidad de elección con una gama mayor de cursos en oferta para su matrícula.

Cuadro No. 7 Ingresos propios por periodo académico (en miles de colones) (19941999)

\begin{tabular}{|l|ccc|}
\hline $\begin{array}{c}\text { Periodo } \\
\text { Acodemico }\end{array}$ & $\begin{array}{c}\text { Ingresos } \\
\text { propios }\end{array}$ & $\begin{array}{c}\text { Ingresos } \\
\text { totoles }\end{array}$ & $\Delta \%$ \\
\hline 1994 & $454,805.28$ & $1,857,570.03$ & 24.5 \\
\hline 1995 & $538,927.16$ & $2,283,719.73$ & 23.6 \\
\hline 1996 & $701,464.29$ & $2,768,175.15$ & 25.3 \\
\hline 1997 & $915,162.96$ & $3,405,891.79$ & 26.9 \\
\hline 1998 & $1,212,231.29$ & $4,142,060.62$ & 29.3 \\
\hline
\end{tabular}

Fuente: Anuarios Estadísticos 1994-1997

En relación con el tercer objetivo, se observa que los ingresos propios presentan un crecimiento bastante regular durante el período de estudio. A partir de 1996, se dan 
incrementos graduales de 1 a 2 puntos porcentuales. Esto permite concluir que la composición de ingresos propios versus ingresos totales no tuvo alteraciones importantes con la entrada de la cuatrimestralización. A lo largo de este documento, se insiste en la necesidad de revisar la oferta académica, de manera que ofrezca una gama mayor de asignaturas que facilite el progreso de la carrera en cada periodo académico, y no solo en forma anual; esta situación, en definitiva, puede estar causando una distribución menos marcada hacia la autogeneración de recursos.

\subsection{MEJOR APROVECHAMIENTO DE LOS RECURSOS DE LA UNIVERSIDAD}

Este objetivo se relaciona con la asignación y aprovechamiento de los recursos humanos, físicos, tecnológicos y financieros en los distintos programas académicos en oferta. La modalidad cuatrimestral genera la expectativa de una mejor distribución y utilización de estos recursos. En el presente informe esta valoración no fue considerada, debido a su magnitud y a la poca disponibilidad de tiempo para realizar este análisis, que por sí mismo, justifica una evaluación aparte.

\section{IMPLICACIONES ADICIONALES EN LOS RESULTADOS ACADÉMICOS DEL ESTUDIANTE}

El acuerdo tomado por el Consejo Universitario, sesión No. 1171-95 Art. II, inciso 2, se manifiesta como una oportunidad a la innovación: "la cuatrimestra. lización no debe limitarse solo a periodos más cortos, sino también a formas novedosas de entrega de la docencia, de evaluación de los aprendizajes y de acreditación por experiencia". Es por eso por lo que, a partir de esta sección del documento, se analizan distintos aspectos que pueden reflejar algún tipo de consecuencia que se encuentre inscrito o no, en el sentido de esta directriz y que sea conveniente identificar para así poder tomar las acciones pertinentes que coadyuven en alguna medida a solventar los problemas que de ahí se derivan.

De esta forma, se incorporan al estudio el comportamiento de otras series estadísticas que puedan dar luz sobre las posibles repercusiones en la actividad académica, así, se analizan también las apelaciones y la deserción por periodo académico.

Comportamiento de las apelaciones

En el cuadro No. 8 se observa un porcentaje de las materias en oferta en 1996 que se limita la evaluación del estudiante a solo exámenes, según la planificación que aparece en el Proyecto Cuatrimestralización (1996). Algunas de estas asignaturas se vieron en la necesidad de eliminar las tareas, los estudios de casos o los proyectos que se practicaban bajo la modalidad semestral para poder ajustarse así a la disminución del tiempo para la evaluación de los aprendizajes.

Esta situación puede limitar las posibilidades de asimilación y la capacidad de aplicación adecuada de los conocimientos disciplinarios en materias de naturaleza teórico-práctica, pues precisamente se discriminan como procedimiento evaluativos los trabajos más prácticos y complejos a favor de procedimientos mucho más simples cuales son los exámenes con un porcentaje alto de preguntas objetivas; ello, dado que 
estos últimos exigen una menor dedicación en su planeamiento yen su corrección. También, la evaluación formativa resulta cada día más difícil de efectuar particularmente en cursos numerosos. Esta situación se debe a lo comprensivo de los tiempos entre una actividad evaluativa y la otra y al exiguo tiempo asignado para la corrección de los instrumentos evaluativos (en el reglamento se asignan 5 minutos máximo por examen). Esta situación se complica aún más en los cursos que se les redujo la asignación de tutores.

Es notable en dicho cuadro que la escuela de Ciencias Exactas y Naturales tiene un comportamiento diferente de la evaluación de aprendizajes, este puede estar causado por la naturaleza aplicada de estas disciplinas o por una mejor relación personal académicoestudiante. De ahí que sea conveniente que se realice un análisis cualitativo en todos los cursos que ofrece la UNED, estableciendo prioridad en las materias ejes de las carreras. En la sección se hace un esfuerzo inicial por conocer esta situación para cuatro ejes de la carrera de Ciencias de la Educación de 1 y II ciclos.

Cuadro No. 8 No. de materias con evaluación de los aprendizajes basada en exámenes por escuela en 1996

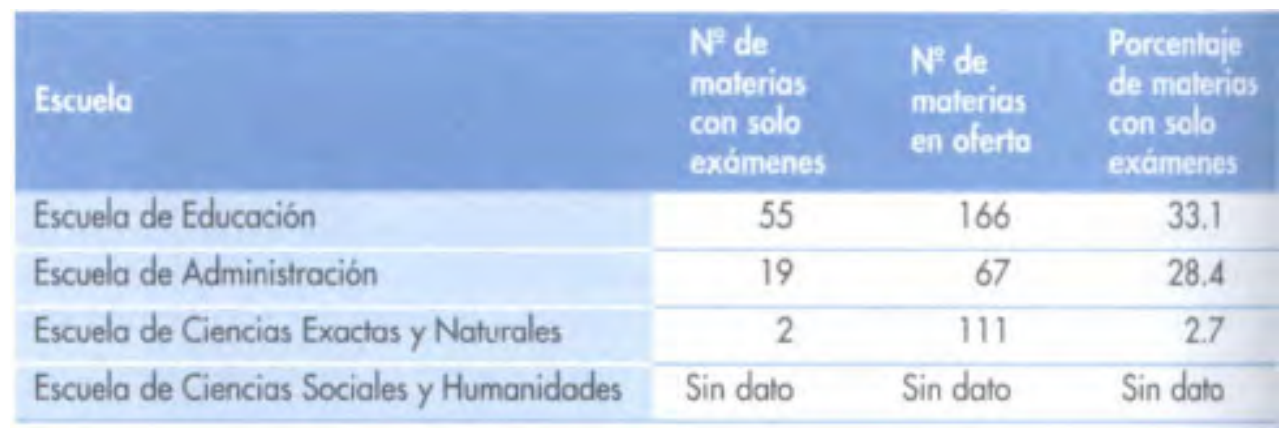

Fuente: Proyecto de cuatrimestralizoción 1996

Es de suponer que al haber menos tiempo para la calificación de estos exámenes, es de esperar que surjan más errores en la corrección de estos instrumentos y esto a su vez produzca una mayor incidencia de apelaciones de notas por parte de los estudiantes; de ahí que, se considera importante incorporar st variable en la evaluación.

En el cuadro No. 9 se incluye el número de apelaciones promedio por ma ria ofrecida en los respectivos periodos académicos, la información que se obt ne para los dos primeros años del estudio tiene cierto grado de subestimación q dificulta valorar con precisión el impacto que pudo tener la cuatrimestralizaci en este componente del servicio estudiantil. Pese a lo anterior, el nivel en 1996 similar al del 1a semestre de 1994; de ahí que se puede concluir que la entrada la cuatrimestralización no provoca incrementos significativos en la anelacio de notas: recuérdese que este último dato está subestimado.

Para los años siguientes, se observa alguna irregularidad en el cor miento por cuatrimestre, y en los dos últimos, se nota una tendencia a la uniformidad, en un promedio de poco más de 3 apelaciones por asignatura, a pesar de que en esos años fue justamente cuando de nuevo se amplió la oferta académica.

Esto permite concluir que, los reclamos hechos por los estudiantes debido a posibles errores cometidos en la calificación de sus exámenes no se han visto muy afectados por 
la cuatrimestralización: más bien, existe una tendencia a mantener el nivel bajo control dentro de un rango reducido. Un factor que puede estar favoreciendo este resultado es el esfuerzo que se viene realizando para eliminar las inconsistencias en la confección de estos instrumentos evaluativos y la presencia del alto porcentaje de preguntas objetivas que se incorporan en los exámenes. Y así, este rango se encuentra dentro de 1 a 6 apelaciones en promedio por materia para el periodo estudiado, y de 2 a 4 de ellas a partir de 1998

Cuadro No. 9 No. de apelaciones por escuela (1994-1999)

\begin{tabular}{|c|c|c|}
\hline $\begin{array}{l}\text { Periodo } \\
\text { Acodémico }\end{array}$ & Total & $\begin{array}{l}\text { Promedio } \\
\text { por moteria }\end{array}$ \\
\hline $94.1^{\circ}$ & 1497 & 4.20 \\
\hline $94-2^{*}$ & 529 & 1.63 \\
\hline $95-1$ & Sin dato & \\
\hline $95-2^{*}$ & 660 & 1.97 \\
\hline $96-1$ & 1059 & 4.19 \\
\hline $96 \cdot 2$ & 956 & 4.88 \\
\hline $96 \cdot 3$ & 837 & 4.19 \\
\hline $97-1$ & 757 & 2.95 \\
\hline $97-2$ & 1510 & 5.53 \\
\hline $97-3$ & 628 & 2.30 \\
\hline $98-1$ & 975 & 3.04 \\
\hline $98-2$ & 1434 & 3.57 \\
\hline $98-3$ & 1167 & 3.03 \\
\hline 99.1 & 906 & 2.32 \\
\hline $99-2$ & 1384 & \\
\hline
\end{tabular}

* Dato parcial

Fuente: Oficina de Operaciones

Comportamiento de la deserción por periodo académico

La deserción global por periodo académico no muestra diferencias entre el sistema semestral y cuatrimestral: en ambos sistemas se mantiene dentro del intervalo 19 al $25 \%$, con excepción del primer semestre de 1994, que tiene un comportamiento relativamente más favorable del $16 \%$. Estos resultados permiten concluir que la cuatrimestralización no promovió una mayor deserción entre los estudiantes; un factor que favorece esta situación se atribuye posiblemente a que el estudiante hace una mejor distribución de la carga académica frente a la oportunidad de un periodo lectivo adicional en el año. 
Cuadro No. 10

\begin{tabular}{|l|l|}
\hline $\begin{array}{l}\text { Periodo } \\
\text { Acodémico }\end{array}$ & $\begin{array}{l}\text { Porcentaje } \\
\text { de deserción }\end{array}$ \\
\hline $1994-1$ & 15.5 \\
\hline $1994-2$ & 23.9 \\
\hline $1995-1$ & 23.3 \\
\hline $1995-2$ & 24.2 \\
\hline $1996-1$ & 22.5 \\
\hline $1996-2$ & 24.4 \\
\hline $1996-3$ & 18.6 \\
\hline $1997-1$ & 22.3 \\
\hline $1997-2$ & 18.9 \\
\hline $1997-3$ & 19.6 \\
\hline $1998-1$ & 20.6 \\
\hline $1998-2$ & 20.0 \\
\hline $1998-3$ & 21.3 \\
\hline $1999-1$ & 22.0 \\
\hline $1999-2$ & 18.5 \\
\hline
\end{tabular}

* Se contabilizan el retiro ¡ustificado, retiro autorizado, no se presentó (NSP) Fuente: Ofkina de Registro, octubre 1999

Análisis de trayectoria de las series estadísticas consideradas

A manera de síntesis, se propone un modelo relacional multivariable de algunas series númericas que se contemplan en este estudio, a fin de lograr una primera aproximación a un análisis de vías causales que sirva de base para posteriores estudios y que contribuya a la comprensión del comportamiento de estas variables académicas claves y así poder tomar decisiones que favorezcan las expectativas del proceso cuatrimestral.

Análisis de trayectoria del proceso cuatrimestral

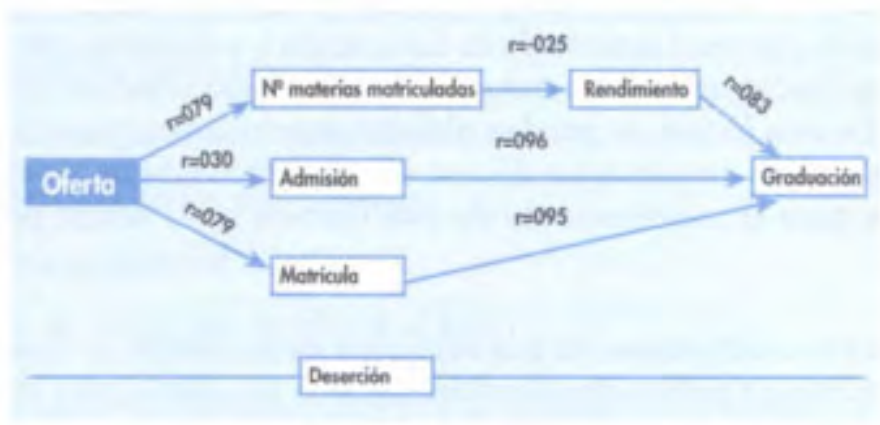

De acuerdo con los resultados del análisis de trayectoria propuesto, se determina la influencia que tiene la oferta académica (variable exógena) en la respuesta del 
estudiante en cuanto a las materias que decide matricular en cada periodo académico, y de ahí sus efectos en el rendimiento (variable endógena), que como es de esperar presenta una correlación negativa; es decir, cuantas más materias matricule, su rendimiento tiende a disminuir $(\mathrm{r}=-\mathrm{O} .25)$. Esta situación como se muestra en la primera vía definida tiene implicaciones más tarde, en las posibilidades de graduación del estudiante (variable endógena). Asimismo, se puede concluir que en las otras dos trayectorias consideradas, la admisión y la matrícula se ven también influenciados por la disponibilidad de materias a matricular, y esta situación tarde o temprano favorece o perjudica los resultados que se obtengan en la graduación de estudiantes. Por otra parte, la deserción tiene un comportamiento independiente a estas variables académicas. Posiblemente, se recuerda que ésta no tiene alteraciones con la nueva modalidad.

Este análisis permite reafirmar el punto mencionado sobre la relevancia de realizar un planeamiento cuidadoso de la oferta académica, pues tiene implicaciones como las indicadas en el párrafo anterior, y tambíen en la motivación del estudiante sobre las expectativas que éste tiene ante la modalidad cuatrimestral.

\section{ANÁLISIS FOCALIZADO: IMPLICACIONES EN LA CARRERA DE CIENCIAS DE LA EDUCACIÓN 1 Y II CICLOS Y SUS EFECTOS EN ALGUNOS CURSOS EJES.}

Debido a la conveniencia de completar este análisis cuantitativo con elementos cualitativos de interés académico, se han seleccionado cuatro ejes pro- gramáticos de la carrera Ciencias de la Educación 1 y II ciclos que permitan conocer las implicaciones de la reducción del periodo lectivo en esos casos particulares. De esta forma, se pueden obtener conclusiones generales a estos ejes y particulares a cada uno de acuerdo con su especificidad que puedan ser tomadas en cuenta para el mejoramiento de esta carrera en el actual proceso cuatrimestral.

Los criterios establecidos para la selección de la carrera se basan en que ésta concentra la mayor población estudiantil y, a su vez, tiene gran importancia en el desarrollo social del país. Para la elección de las asignaturas, se utilizan los mismos argumentos, de manera que se eligen algunas materias claves en la formación del estudiante: así, se analizan los cursos de Planeamiento didáctico, Di. dáctica del español, Didáctica de las ciencias naturales y una asignatura de corte aplicado que se encuentra al final del programa de diplomado que es la Práctica docente.

A nivel general, un aspecto que se relaciona con cada uno de estos ejes, y en realidad con cualquier materia, es que previo a su revisión específica se requiere efectuar una revisión de la carrera como tal. Antes de adaptar el curso en sus contenidos y en su enfoque didáctico se debe reorientar el plan de estudios de la carrera como un todo. Así que, una vista parcial del contexto curricular global, como es efectivamente la adaptación de una materia aislada, corre el riesgo de afectar la coherencia interna del plan de estudios y con ello desviar el perfil de salida previsto. De esta forma, pueden dejar inhabilitados enlaces cognoscitivos entre materias relacionadas, que alteran la continuidad del conocimiento y limitan el desarrollo de destrezas, habilidades y actitudes, y por ende, producen un vacío que puede afectar el desempeño profesional. A su vez, esta situación debe ser vista como una oportunidad para la actualización del 
programa académico que valore su pertinencia y relevancia acorde con las exigencias de los nuevos tiempos.

La experiencia valiosa que se ha adquirido a la fecha permite realizar una revisión cuidadosa de los planes de estudio, hacer modificaciones graduales, y con ellas, incorporar ajustes en los cursos claves para la formación de las competencias profesionales. Esta labor también está enmarcada en la tendencia actual de mejoramiento con miras a la acreditación, respecto a la cual la Universidad está comprometida.

Análisis en cada curso eje seleccionado

Ahora bien, para la valoración de las implicaciones académicas en estos cuatro ejes se consideraron los aspectos que se indican abajo, desde la perspectiva de los encargados de cátedra y de la encargada del programa académico. Para estudios posteriores, conviene triangular esta información con otros sectores involucrados, así como con el criterio de expertos en la temática:

- La adaptación curricular del curso

- La actualización del material didáctico La evaluación de los aprendizajes

- La tutoría presencial

- Número de veces que se ofrece al año

Entonces, ¿qué tipo de adaptaciones se realiza? Al respecto, se reitera la viSión micro empleada para efectuar tales ajustes, en vista de que se estudia el curso aislado del contexto del programa de la carrera. En general se observa que se dejan los mismos contenidos y se hacen distintas adaptaciones de acuerdo con la naturaleza del curso; en algunos casos se cambian los objetivos: de más específicos a más generales; en otros, los temas se concentran o se hacen en forma simultánea y se eliminan las actividades formativas tales como elaboración de las tareas para dejar la evaluación de los aprendizajes en la aplicación de exámenes.

Estos ejes tienen como denominador común que son de naturaleza teórico- práctica: es decir, la aplicación de los conocimientos es básica para lograr la comprensión adecuada de sus contenidos. Además, en ellos se atienden una gran cantidad de estudiantes en cada periodo académico. La evaluación se basa en dos exámenes ordinarios, las tareas se eliminan, y la práctica se incorpora entonces con preguntas de desarrollo en los instrumentos evaluativos. La asistencia tutorial coordinada por la cátedra es solo parcial en la Región Central (San José, Alajuela, Cartago y Heredia), mientras que el resto lo administra el centro universitario en forma independiente. El número de tutorías se redujo a la mitad por el recorte en la asignación de tutores y por la compresión del periodo lectivo. Actualmente, existe una carga excesiva en la evaluación de instrumentos por tutor evaluador; éstos deben corregir entre 200 y 400 instrumentos en 6 o 8 días calendario. Entonces, existe una reducción de los servicios de apoyo. La oferta académica se mantiene similar al sistema anterior, puesto que solo se ofrece dos veces al año. 
En cuanto a las adaptaciones curriculares del curso, se mantienen los mismos contenidos del sistema semestral, pero se cambió o se está en proceso de cambio de la unidad didáctica, la cual se adapta a los nuevos enfoques pedagógicos y se ajusta a la disponibilidad de tiempo en la nueva modalidad.

Esta situación difiere en la Práctica Docente que por su naturaleza aplicada las actividades de aprendizaje y de evaluación se realizan en una escuela primaria y responde a una programación educativa preestablecida, a cargo de un supervisor contratado por la UNED y se colabora dentro de una aula escolar, en asistencia a una maestra de grado escolar.

Con respecto a lo observado en los cuatro cursos, se puede concluir que para lograr un proceso cuatrimestral más positivo desde el punto de vista académico es oportuno revisar el plan de estudios y el curriculum de cada curso y posteriormente, el paquete instruccional. Se nota también que la transformación de la modalidad afecta de manera particular la práctica y los cursos teórico-prácticos. Para ello se recomienda revisar las actividades de aprendizaje y las evaluativas, así como hacer una asignación racional de los recursos humanos, tecnológicos, físicos y financieros para que se puedan introducir los ajustes que requieren estas materias.

\section{RECOMENDACIONES PARA LA CARRERA DE CIENCIAS DE LA EDUCACIÓN EN I Y II CICLOS DESDE LA ÓPTICA DE CUATRO DE SUS EJES}

Luego de una revisión sobre aspectos básicos en los cuatro cursos descritos de la carrera Ciencias de la Educación en 1 y II ciclos, se exponen algunas sugerencias en el sentido de solventar situaciones de nivel académico que están presentes todavía y que son susceptibles de resolver a un corto plazo. Fundamentalmente, se plantean algunas ideas que implican la realización de diversos proyectos administrativos, de investigación, de planeamiento, que son competencia de diversas instancias institucionales que mucho tienen que aportar para el logro de un proceso de cuatrimestralización expedito, seguro, y eficaz. De seguido se indican las sugerencias particulares para la carrera elegida.

- Hacer una revisión del plan de estudio de la carrera con propósitos de comprobar la pertinencia, la relevancia y la coherencia de su curriculum y de sus actividades de aprendizaje, y de ajustarla a las condiciones de la modalidad cuatrimestral.

- Realizar un estudio de caracterización de la población meta de la carrera que permita conocer sus condiciones ambientales y académicas para así poder dirigir el esfuerzo educativo enfocado a sus posibilidades particulares. De esta forma, mejorar la comunicación didáctica, promover la aplicación de conocimientos, tener una relación más personalizada y agilizar la revisión de los proyectos de investigación. $\mathrm{Y}$ en aquellos sectores poblacionales que sea aplicable, poder introducir innovación pedagógica con los recursos modernos de las tecnologías de información. 
- Promover la comunicación entre alumnos, por medio de los ya conocidos círculos de estudios, o en caso de sea posible, por vía de la red Internet, donde la Universidad sirva como canal de comunicación para su conformación, su orientación evaluación.

- Facilitar un medio bidireccional personal o mediatizado para atender sus consultas dado que el material impreso y audiovisual de carácter unidireccional, no pueden prever todas las dudas que surgen en un ambiente de autoestudio. La supresión de tutorías puede afectar en este aspecto, especialmente, en los cursos de naturaleza práctica. Por otro lado, el contacto personal con el sistema educativo es importante en la formación integral de estudiante.

- La carga académica y administrativa adicional que significa la cuatrimestralización resulta difícil de administrar, más aún, cuando se atienden una gran cantidad de estudiantes como es el caso analizado. De ahí que, en aras de no afectar el servicio al estudiante, conviene revisar la asignación de tutores tanto para el servicio presencial, como para la evaluación de los aprendizajes.

- Incorporar prácticas en los cursos que así lo requieran, uno de los factores que más afecta es sin duda la gran cantidad de estudiantes matriculados, que dificulta la calificación en esos tiempos tan reducidos y la comprobación de la autoría del trabajo práctico; sin embargo, esta situación puede ser solventada, por ahora, con la asignación de más apoyo de personal académico, para no convertir los cursos básicos para la formación profesional en tratamientos educativos que solo apropien conocimientos pero sean débiles en la generación de las competencias disciplinarias integrales.

- Poner en práctica un proyecto piloto en el curso de Práctica docente, que cuente con la infraestructura tecnológica y humana requerida en la misma área de trabajo donde se administra la carrera. Así, las actividades de revisión de el diagnóstico, el proyecto educativo, el planeamiento y la asesoría la realice un supervisor del área disciplinaria que esté capacitado en el manejo de herramientas informáticas. Esta propuesta tiene proyección para otros cursos y para mejorar la comunicación con los estudiantes. En otras palabras, se abre un camino para nuevas experiencias que permitan modernizar los recursos disponibles y fortalecer con el proyecto tecnológico- educativo de la Institución un curso clave de una carrera relevante.

- Ofrecer más apoyo administrativo y tecnológico a la carrera de Ciencias de la Educación de 1 y II ciclos, que permita un servicio ágil de atención a los estudiantes. Es importante también revisar los procesos administrativos que logren ser expeditos sin perder confiabilidad y con apoyo logístico y tecnológico suficiente.

- Ampliar la Práctica Docente a un semestre, o en su defecto, solo ofrecerla en los dos últimos cuatrimestres, debido a que el estudiante realmente pierde la oportunidad de aplicar los conocimientos fundamentales de su formación a nivel de diplomado, por el escaso tiempo disponible para tal actividad. 


\section{CONCLUSIONES Y RECOMENDACIONES GENERALES}

En esta última sección se presentan las conclusiones y recomendaciones de carácter general derivadas de la exposición antecedente y se finaliza con la identificación de algunos proyectos que incorporan las sugerencias propuestas.

\section{Conclusiones}

En relación con el cumplimiento de los objetivos, se puede concluir que:

- Las expectativas sobre un avance más rápido del programa de estudios y por ende, de una mejor posibilidad de acortar el tiempo de inserción en el mercado laboral, de una mayor población de estudiantes nuevos y antiguos anual, se ve efectivamente favorecida. Ahora bien, no fue posible deducir si esta agilidad académica también favorece la absorción de estudiantes, porque estos ven con muy buenos ojos la oportunidad de una graduación a más corto plazo, puesto que los incrementos observados están asociados al periodo académico adicional, en condiciones que se perfilan, para esa época del año, como la única opción de estudio en una institución de educación superior estatal. También se observa un aumento en el rendimiento académico general, no obstante, es importante anotar que, un mejor rendimiento estudiantil no implica, necesariamente, una mayor calidad en la preparación profesional, sobre todo, si existen limitaciones en las actividades de aprendizaje y los servicios de apoyo que requiere la carrera. Los graduados tienen un aumento notable de poco más del doble anual; esto reafinna el cumplimiento de este primer objetivo. No obstante, la flexibilidad de los requisitos para realizar el trabajo final de graduación, sin duda, fue un factor que tiene repercusión en este resultado. ¿Cuál sería el comportamiento si se hubiesen mantenido los mismos requisitos de graduación? Por otro lado, la transformación propuesta a raíz de la cuatrimestralización tiene un sentido positivo y eventualmente, puede ser vista como una oportunidad de acortar el tiempo de graduación; esto es así, en la medida de que las posibilidades académicas y económicas del estudiante se lo permitan. La UNED por su parte, requiere que esta oportunidad sea real, por medio de una oferta racional que permita tal progreso al ritmo que las posibilidades personales de cada alumno.

- La posibilidad planteada de una mayor circulación del material didáctico se puede inferir que se cumple si se deduce del efecto que puede tener el incremento de la matrícula y admisión anual; no obstante, en aquellas materias que se reduce o mantienen la oferta, es de esperar que esta situación no se presente. Por otra parte, la afirmación de que esta mayor rotación favorece la actualización del material didáctico, solo puede ser parcialmente estudiada a la luz de los cuatro ejes seleccionados. Al respecto, se observa que el material didáctico ya se había cambiado en uno de estos cursos, y en los otros, se encuentra en proceso la actualización; aunque esta información es insuficiente para una generalización, en estos casos particulares se nota que la cuatrimestralización pudo haber favorecido este cambio en el material de estudio. 
- En cuanto a un mejor aprovechamiento de los recursos, y pese a que, antes se indicó que este aspecto no es analizado en el presente informe, sí se puede percibir que el personal académico incrementa su carga en actividades docentes (tutorías, elaboración y calificación de exámenes) o administrativo-docente (cuido de exámenes, atención de estudiantes). Y en este punto, se refiere el análisis cualitativo mencionado al principio (Cruz, 1999), donde los docentes coinciden en que la cuatrimestralización ha generado un cansancio excesivo por el incremento en la atención de la docencia. Además, esta situación se intensifica con el recorte de recursos de tutoría realizado desde el inicio de esta modalidad. Esto no significa necesariamente que exista una mayor oportunidad para realizar actividades de otro tipo de desarrollo académico, ligados a la investigación, la producción de material didáctico, o la actualización profesional, o la actualización del curriculum de la carrera o de los cursos; más bien, pareciera ser lo contrario. No obstante, este aspecto no fue analizado, pero por su importancia en la calidad académica y en la motivación profesional conviene también se preste atención en futuros estudios. La pregunta que surge ahora es ¿cuánto se pudo haber afectado estas actividades de gran interés académico por la atención de las otras de carácter docente o administrativo-docente mencionadas?.

- En cuanto a la referencia de ingresos propios, se nota que los incrementos en la composición de ingresos totales no varía significativamente. Por otro lado, el comportamiento de los costos no es posible incorporarlos en este estudio por las razones ya expuestas. Pero si la Universidad desea ampliar este concepto, debe ir ligado a acciones institucionales que favorezcan esta composición, y uno de ellos es un planeamiento adecuado de la oferta académica

\section{Recomendaciones}

Si efectivamente, la cuatrimestralización cumplió con las expectativas principales, es decir, se favoreció entre otras cosas, la duración de las carreras, la rotación de material didáctico, el rendimiento ante una mejor distribución de la carga académica y la utilización de recursos, se debe concluir que ¿solo implicaciones positivas genera esta nueva modalidad?.

Pareciera que se dieron consecuencias no previstas en su gestación que coinciden con las conclusiones a que llegaron los académicos de la Universidad de Costa Rica hace más de 20 años; así, se observa que, a nivel cualitativo, los cursos tuvieron que sacrificar sus actividades de aprendizaje formativos, los profesores vieron incrementada su carga académica en actividades limitadas a las tutorías, la elaboración, la calificación y el cuido de exámenes y a la atención de los estudiantes, puesto que estas actividades se vieron intensificadas con un nuevo periodo académico y a la reducción de los recursos para brindar este servicio de apoyo. Así mismo, es de comprender que estas actividades pueden resultar en exceso poco motivadoras.

Al respecto, se refiere el estudio "Propuesta para elaborar el programa de administración de empresas", donde, entre otros temas, se analiza la carga académica de los profesores de la escuela de Ciencias de la Administración en el II cuatrimestre de 1997. En esta misma sección: "se observa que el porcentaje de tiempo dedicado a las denominadas actividades académico-administrativas es, en algunos casos, mayor a las denominadas académicas. Aunque esta situación señalada se refiere a una escuela en 
particular, es de suponer que estas condiciones se estén presentando en las otras escuelas, por lo que conviene hacer una revisión de esta carga académica, de manera que permita "el desarrollo de proyectos de investigación que le permitan la retroalimentación y la actualización en el área de la especialidad, así como mantener un vínculo con su quehacer docente y de esta manera lograr un nexo entre la docencia, la investigación y la extensión.” (Cruz, Hidalgo, Castillo, 1997, p. 69-75).

De seguido se describen las recomendaciones:

- Hacer una revisión de los planes de estudio de las carreras y un estudio focalizado en los ejes programáticos prioritariamente. Analizar los problemas y determinar las soluciones a éstos. Revisar la actualización del programa académico, los contenidos, las actividades y la evaluación de los aprendizajes de $\operatorname{los}$

cursos.

- Orientar el desarrollo teórico-práctico en los cursos claves de la formación profesional. En los casos donde se necesite realizar actividades y evaluación de los aprendizajes, asignar mayor tiempo a la realización y revisión de las pruebas que la asignada en el reglamento de asignación de cargas académicas vigente. Este tiempo designado puede ser suficiente en algunos casos donde prevalezca la pregunta cerrada o de respuesta breve, pero en preguntas de desarrollo, estudios de casos, proyectos, entre otros, no es posible logra una evaluación adecuada en ese tiempo previsto.

- Definir lineamientos y criterios para orientar las actividades de investigación y evaluación y concentrar los esfuerzos y los recursos institucionales prioritariamente a fortalecer los programas académicos claves. Para ello es necesario definir algunos criterios de selección y priorización. De esta forma tal que, la Universidad estaría principalmente estimulando los proyectos experimentales de innovación tecnológica, de programas de capacitación, de temática en los congresos internacionales, de proyecto de investigación educativa, entre otros puntos de interés, a aquellos que fortalezcan estas áreas institucionales de desarrollo prioritario.

- Realizar un estudio de la situación del mercado laboral para profesionales en educación primaria, sus tendencias y las repercusiones en la competitividad institucional que tiene la incorporación del sector universitario privado.

- Revisar el planteamiento de la oferta académica que considere criterios válidos y racionales que permitan el progreso real de los estudiantes de la carrera.

- Realizar un estudio comparativo de costo-efectividad para las dos modalidades: conviene valorar el aprovechamiento de los recursos financieros, humanos, físicos y tecnológicos utilizados, determinar si estos producen los mismos o mejores resultados, y si éstos costos son iguales o mayores en términos de la productividad obtenida. Aquí, únicamente se plantea la inquietud para que sea atendida estudios. 
- Hacer una revisión de la carga académica que permita a los profesores disfrutar de otras actividades de desarrollo profesional, que en definitiva contribuye a una mejor disposición hacia la actividad docente.

Finalmente, el ensayo se concluye con la identificación y descripción breve de algunos proyectos que se derivan de las recomendaciones anteriores, cuyo planteamiento y desarrollo tiene sus responsables según laestructura organizacional de la UNED:

Cuadro No. 11 Proyectos propuestos para realizar ajuste a la modalidad cuatrimestral

\begin{tabular}{|c|c|c|c|}
\hline & Muy bueno & Bueno Regulor & Deficiente \\
\hline Exactitud y solvencia del material & III & & \\
\hline Estructura y secuencia interna de las actividades & II & 1 & \\
\hline Factibilidod y practicidod de las propuestas & ॥ & 1 & \\
\hline Claridad y significancia & III & & \\
\hline Opinión general del servicio & 1 & ॥ & \\
\hline & Alio & Medio & Bojo \\
\hline Nivel de dificultod hallodo & $20 \%$ & $60 \%$ & $20 \%$ \\
\hline Reloción a sus intereses & $30 \%$ & $60 \%$ & $10 \%$ \\
\hline $\begin{array}{l}\text { Importancia frente a las } \\
\text { necesidodes lecnológicas actuales }\end{array}$ & $20 \%$ & $80 \%$ & \\
\hline Impodo general del material & $30 \%$ & $60 \%$ & $10 \%$ \\
\hline
\end{tabular}

\section{REFERENCIAS BIBLIOGRÁFICAS}

CENTRO DE INVESTIGACIÓN PARA EL DESARROLLO DE LA EDUCACIÓN A DISTANCIA (1994). Anuario estadístico. San José: Universidad Estatal a Distancia. (1995). Anuario estadístico. San José: Universidad Estatal a Distancia.

(1996). Estudio de opinión sobre el proceso de matrícula 1 cuatrimestre. San José: Universidad Estatal a Distancia.

CRUZ, A., HIDALGO, G., CASTILLO, A. (1997). Propuesta para mejorar el programa de administración de empresas. San José: Universidad Estatal a Distancia.

CRUZ, A. (1999). La cuatrimestralización en la UNED. San José: Universidad Estatal a Distancia. 124 Valoración en el cumplimiento de los objetivos en la cuatrimestralización de la UNED.

DRUCKER, P., (1996) Su visión sobre: La administración, la organización basada en la información, la economía y la sociedad. Barcelona: Norma.

ESCOTET, M. (1992) Aprender para el futuro. Madrid: Alianza 
ESCUELA DE CIENCIAS SOCIALES Y HUMANIDADES (1992). Proyecto de cuatrimestralización. San José: Universidad Estatal a Distancia.

HIDALGO, G.( 1999). Evaluación del proceso de cuatrimestralización a la luz de algunas series estadísticas. UNED: documento mimeografiado.

RECTORÍA (1997). Informe de labores 1996. San José: Universidad Estatal a Distancia.

(1997). Informe de labores La UNED hacia el siglo XXI 1986-1996. San José: Universidad Estatal a Distancia.

(1998). Informe de labores 1997. San José: Universidad Estatal a Distancia.

UNED (1995). Proyecto de cuatrimestralización 1996, San José: Universidad Estatal a Distancia.

VICERRECTORIA DE PLANIFICACIÓN (1997). Estadísticas 1992-1996. San José: Universidad Estatal a Distancia.

VICERRECTORIA DE PLANIFICACIÓN (1998). Anuario estadístico 1997. San José: Universidad Estatal a Distancia.

\section{PERFIL ACADÉMICO Y PROFESIONAL DE LA AUTORA}

Guiselle Hidalgo Molina es licenciada en Estadística, con especialización a nivel de posgrado en Informática de la Universidad de Costa Rica, máster en Econometría del Centro de Investigación y Docencia Económicas (CIDE) México D. F. Experiencia docente en la Universidad de Costa Rica, Universidad Nacional de Costa Rica, Universidad Estatal a Distancia de Costa Rica (UNED) y en el CIDE en México D. F. Ha realizado investigaciones en educación, estadística e informática. Además, ha llevado a cabo evaluaciones de programas académicos universitarios en el Centro de Investigación y Evaluación Académica de la UNED. Estuvo en la dirección del Centro de Informática de la UNED durante 10 años, tiene experiencia en proyectos tecnológicos aplicados a la edu. cación a distancia en el campo de la administración estratégica y en la educación superior. Dispone de varias publicaciones en estadistica, educación e informática.

\section{Guiseille Hidalgo Molina}

Centro de Investigación académica (ClAC)

Universidad Estatal a Distancia (UNED)

email: gmolina@uned.ac.cr

$\operatorname{Fax}(506) 2341909$

Telef (506)25321 21 extensión 2242

Apartado 474/2050

San Pedro de Montes de Oca

San José, Costa Rica 